\title{
Milligram per Week
}

National Cancer Institute

\section{Source}

National Cancer Institute. Milligram per Week. NCI Thesaurus. Code C67404.

A dose administration rate unit equal to the rate at which one thousandth of a gram of a product is delivered or administered over the time period of one week. This is also a unit of mass flow rate equivalent to the rate at which one thousandth of a gram of matter crosses a given surface or is delivered to a given object or space over a period of time equal to one week. 